\title{
Thermodynamics of suspensions of polymeric chains in dilute solutions
}

\author{
Annunziata Palumbo, Antonino Valenti and \\ Georgy Lebon
}

\begin{abstract}
A model of suspensions of deformable polymeric chains in dilute solutions is proposed. The originality of the model lies in raising the relative motion of polymers with respect to the solvent to the status of independent variables, at the same level of such classical variables as global mass, mass fraction, temperature, and conformation tensor. Such an attitude is typical of extended irreversible thermodynamics. It is shown that the restrictions placed by the second law of thermodynamics leads to the various expressions of the constitutive and evolution equations governing the model. The use of Onsager's reciprocal relations and the criterion of frame indifference is also commented upon.
\end{abstract}

\section{Introduction}

The object of this work is to propose a thermodynamic formulation of suspended deformable macromolecules in motion with respect to a solvent carrier. The driven particles are supposed to be large chains of polymers whose shape is described by the second-order symmetric conformation tensor $\mathbf{C}=\int \mathbf{r r} \psi(\mathbf{r}) d \mathbf{r}$, where $\mathbf{r}$ is the end-to-end macromolecular distance vector and $\psi(\mathbf{r})$ the distribution function. The problem is of wide practical interest, in particular to better comprehend the processes of diffusion and migration of polymers. It is observed that macromolecules moving in a non-homogeneous flow tend to migrate to the less inhomogeneous region. Connected problems are the motion of macro particles toward regions of higher stresses or the anomalous retention of polymers in porous media.

Problems involving suspensions are generally treated within the framework of internal variable theories (e.g., $[6,14,15,17])$. The originality of the 
present approach is the reference to extended irreversible thermodynamics (EIT) (e.g., [10,12]). In this theory, the dissipation fluxes like the heat flux and the drift flow of particles are elevated to the status of independent variables, at the same level as such classical variables like mass, momentum, and temperature. One main objective in this paper is to derive the evolution equation of the matter flow, which should boil down to Fick's law as a limit case. Another important parameter is the conformation stress, considered as internal variable and whose behavior in the course of time and space must also be derived in compatibility with the structure of EIT.

The paper will be organized as follows: The space of state variables is defined in Section 2 and consists in the union of the space of classical, internal, and flux variables. Formulating the evolution equations of the classical state variables does not raise any problem and is achieved in Section 3, where, before establishing the evolution equations of the flux and internal variables, we recall the main ingredients of EIT and, after writing the corresponding Gibbs equation, we examine the restrictions placed by the second law of thermodynamics on the form of the evolution and constitutive relations. Comments on the form of the evolution and constitutive equations are displayed in Section 4. Comparison with other approaches and general conclusions are found in Section 5.

\section{State variables and field equations}

The polymeric solution is modeled as a binary mixture with the solvent identified as the carrier fluid and the macro polymeric molecules constituting the dispersed phase. Let $\rho_{\mathrm{c}}$ and $\rho_{\mathrm{p}}$ designate the mass per unit volume of the carrier and the polymeric chains respectively, the total mass density is given by

$$
\rho=\phi \rho_{\mathrm{p}}+(1-\phi) \rho_{\mathrm{c}}
$$

with $\phi$ denoting the volume fraction of the suspended molecules. In view of future developments, let us also introduce the polymeric mass fraction $c$ by $\rho c=\phi \rho_{\mathrm{p}}$. Because of the large inertia of the polymeric chains, the average velocities of the macromolecules $\mathbf{v}_{\mathrm{p}}$ and fluid carrier $\mathbf{v}_{\mathrm{c}}$ are rather different, whence the importance of the particles' diffusion flux

$$
\mathbf{J}=\rho c(1-c)\left(\mathbf{v}_{\mathrm{p}}-\mathbf{v}_{\mathrm{c}}\right)=\rho c\left(\mathbf{v}_{\mathrm{p}}-\mathbf{v}\right),
$$

with $\mathbf{v}$ denoting the mass-weighted velocity

$$
\mathbf{v}=c \mathbf{v}_{\mathrm{p}}+(1-c) \mathbf{v}_{\mathrm{c}},
$$


not to be confused with the volume-weighted velocity

$$
\mathbf{u}=\phi \mathbf{v}_{\mathrm{p}}+(1-\phi) \mathbf{v}_{\mathrm{c}}
$$

For simplicity, indices $p$ of $c, \phi$ and $\mathbf{J}$ have been omitted. Finally, the conformation tensor $\mathbf{C}$ will be assigned to the disperse phase to take the polymer's deformations into account. The space $\mathrm{V}$ of the state variables is formed by the quantities $u, \rho_{\mathrm{p}}, \rho_{\mathrm{c}}, \mathbf{v}_{\mathrm{p}}, \mathbf{v}_{\mathrm{c}}, \mathbf{C}$, where $u$ denotes the specific internal energy coupling with the thermodynamic interactions. However, a better choice of the state variables is the following: $\mathrm{V} \equiv\{\rho, c, T, \mathbf{v}, \mathbf{C}, \mathbf{J}\}$, where $\rho$ and $c$ replace the variables $\rho_{\mathrm{p}}$ and $\rho_{\mathrm{c}}$, respectively, and $\mathbf{v}$ and $\mathbf{J}$ are preferred to the couple $\mathbf{v}_{\mathrm{p}}, \mathbf{v}_{\mathrm{c}} ; T$ stands for the temperature, not necessarily uniform, but assumed to be the same for the solvent and the suspension. Equality of temperatures is justified as thermal exchanges take place at a shorter time scale than momentum and matter exchanges. The reason for the last choice is that most of these variables are more accessible to experience and governed by simpler evolution equations; moreover, the selection of the flux of matter $\mathbf{J}$ as state variable is an attitude commonly adopted in the framework of extended irreversible thermodynamics. Indeed, in this formalism, the flux variables, like the fluxes of matter of momentum (the stress tensor) and energy (the heat flux) are generally upgraded to the rank of state variables at the same level as the "classical" set of variables constituted by the mass, momentum, and energy. In the present work, it is assumed that the relaxation times of the momentum flux (i.e., the stress tensor) and the heat flux are negligibly small compared to the relaxation time of the flux of matter, which is a reasonable assumption in problems of diffusion and suspensions as the ratios of the corresponding relaxation times is of the order of $10^{-3}$ to $10^{-5}$. A more complete treatment in the case of suspensions of rigid particles wherein not only the flux of matter but also the heat flux is used as state variable, can be found in Lebon et al. [13]. Other extended thermodynamics descriptions of complex fluids with internal structures can be found in the book by Jou et al. [9] and a recent work by Manero et al. [18]. The total density $\rho$, mass fraction $c$, momentum $\rho \mathbf{v}$, and internal energy $u$ obey the usual balance laws:

$$
\begin{aligned}
\frac{d \rho}{d t} & =-\rho \nabla \cdot \mathbf{v}, \\
\rho \frac{d c}{d t} & =-\nabla \cdot \mathbf{J},
\end{aligned}
$$




$$
\begin{aligned}
& \rho \frac{d \mathbf{v}}{d t}=-\nabla \cdot \boldsymbol{\Pi}+\rho \mathbf{g}, \\
& \rho \frac{d u}{d t}=-\nabla \cdot \mathbf{q}-\boldsymbol{\Pi}: \nabla \mathbf{v},
\end{aligned}
$$

where $d / d t \equiv \partial / \partial t+\mathbf{v} \cdot \nabla$ is the material time derivative, $\boldsymbol{\Pi}$ is the total stress tensor, $\mathbf{g}$ the acceleration of gravity, and $\mathbf{q}$ the energy flux, a central dot stands for the scalar product and a colon for the double scalar product. The evolution equation for the conformation tensor $\mathbf{C}$ is given in the general form

$$
\frac{d^{*} \mathbf{C}}{d t}=\mathbf{R}(\rho, c, T, \mathbf{D}, \mathbf{C}, \mathbf{J}),
$$

wherein the symmetric velocity gradient tensor $\mathbf{D}=(\nabla \mathbf{v})^{\mathrm{sym}}$ has been selected instead of the velocity field $\mathbf{v}$, to comply with the criterion of material frame indifference (e.g., [21]). To comply with the same requirement, the material derivative in Eq. (9) has been replaced by the Jaumann objective time derivative

$$
\frac{d^{*} \mathbf{C}}{d t}=\frac{d \mathbf{C}}{d t}-\mathbf{C} \cdot \mathbf{W}+\mathbf{W} \cdot \mathbf{C},
$$

wherein $\mathbf{W}$ designates the skew-symmetric velocity gradient. To close the description, one needs constitutive equation for $\boldsymbol{\Pi}, \mathbf{q}$, and $\mathbf{R}$. The latter will be given in the next section. Moreover the evolution equation for the matter flow $\mathbf{J}$ is also missing and will be established in the foregoing as a consequence of restrictions imposed by the second law of thermodynamics.

\section{Thermodynamic analysis}

Since temperature is one of the state variables, it is common to use the Helmholtz free energy $f(\rho, c, T, \mathbf{C}, \mathbf{J})$ as the thermodynamic potential characterizing the system. Developing $f(\rho, c, T, \mathbf{C}, \mathbf{J})$ in Taylor's series around $\mathbf{J}=\mathbf{0}$ and assuming that contributions beyond the second order $J^{2}(=\mathbf{J} \cdot \mathbf{J})$ are negligible, we are allowed to write the specific free-energy in the form

$$
f(\rho, c, T, \mathbf{C}, \mathbf{J})=f_{0}(\rho, c, T, \mathbf{C})+\frac{1}{2} \alpha(\rho, c, T, \mathbf{C}) J^{2},
$$

where sub-index zero refers to a quantity which is $\mathbf{J}$-independent. Thermodynamic stability requires $\alpha$ to be positive because at (local) equilibrium $f$ has a minimum; its dependence on its various arguments is left undetermined at the present stage of the analysis. At the level of the kinetic 
theory, $(1 / 2) \alpha J^{2}$ may be interpreted as taking into account the kinetic energy of diffusion as well as the fluctuating kinetic energies of the solvent and suspended particles (e.g., [14]). The dependence of $\alpha$ with respect to $\mathrm{C}$ reflects the influence of the conformation of the suspended particles on the relative motion between the fluid and the particles; this effect is known as the added mass effect. Under the simplifying hypothesis that $\alpha$ is independent of $\mathbf{C}$ and identified with $1 /\left[\rho^{2} c(1-c)\right]$, the term $(1 / 2) \alpha J^{2}$ represents the kinetic energy of diffusion.

The corresponding differential Gibbs equation, a central quantity in nonequilibrium thermodynamics, is expressed as

$$
d f=-p d v+\mu d c-s d T+\frac{1}{\rho} \mathbf{A}: d \mathbf{C}+\alpha \mathbf{J} \cdot d \mathbf{J},
$$

wherein $v=1 / \rho$ represents the specific volume. The pressure $p$, the specific entropy $s$, the difference between the chemical potentials of the particles and the carrier, $\mu=\mu_{p}-\mu_{c}$, and the tensor $\mathbf{A}$ are defined as:

$$
\begin{aligned}
& p \equiv-f_{v}=p_{0}-\frac{1}{2} \alpha_{v} J^{2}, \\
& s \equiv-f_{T}=s_{0}-\frac{1}{2} \alpha_{T} J^{2}, \\
& \mu \equiv f_{c}=\mu_{0}+\frac{1}{2} \alpha_{c} J^{2}, \\
& \mathbf{A} \equiv \rho f_{\mathbf{C}}=\mathbf{A}_{0}+\frac{1}{2} \rho \alpha_{\mathbf{C}} J^{2},
\end{aligned}
$$

wherein sub-indices in $f$ and $\alpha$ indicate partial derivatives with respect to the corresponding variables, the quantities $p_{0}, s_{0}, \mu_{0}, \mathbf{A}_{0}$ stand for the corresponding derivatives of $f_{0}$; note that although $\alpha$ is a scalar, the quantity $\alpha_{\mathbf{C}}$ represents a second-order tensor which, in the linear approximation, is proportional to $\mathbf{C}$. The tensor $\mathbf{A}$ is symmetric and must vanish at equilibrium; in addition, one must satisfy the property of invariance of $f$ under infinitesimal rotations, implying that $f_{\mathbf{C}} \cdot \mathbf{C}-\mathbf{C} \cdot f_{\mathbf{C}}=0$, from which follows that

$$
\mathbf{A} . \mathbf{C}=\mathbf{C} . \mathbf{A} .
$$

Under the hypothesis that at thermodynamic equilibrium, the suspended molecules are spheres of radius $r_{0}$ and behave like Hookean springs when they are distorted, $\mathbf{A}_{0}$ will take the form [16]

$$
\mathbf{A}_{0}=\frac{3 n k_{0}}{r_{0}^{2}}\left(\mathbf{C}-\mathbf{C}_{\mathrm{eq}}\right), \quad \mathbf{C}_{\mathrm{eq}}=\frac{r_{0}^{2}}{3} \mathbf{I},
$$


where $n$ represents the number of macro-molecules per unit volume, $k_{0}$ a positive elastic constant, and $\mathbf{I}$ the unit tensor.

The second law of thermodynamics expressing the positiveness of the entropy production can be written as

$$
\sigma^{s}=\rho \frac{d s}{d t}+\nabla \cdot \mathbf{J}^{s} \geq 0,
$$

where $\mathbf{J}^{s}$ is the entropy flux and $\sigma^{s}$ the rate of entropy production per unit volume. In terms of Helmholtz's free energy, one has

$$
T \sigma^{s}=\rho \frac{d u}{d t}-\rho \frac{d f}{d t}-\rho s \frac{d T}{d t}+T \nabla \cdot \mathbf{J}^{s} \geq 0 .
$$

Making use of the balance equations (5), (6), and (8) for $\rho, c, u$ and the Gibbs relation (12), one obtains

$$
\begin{aligned}
T \sigma^{s}= & \nabla \cdot\left(T \mathbf{J}^{s}+\mu \mathbf{J}-\mathbf{q}\right)-\mathbf{J}^{s} \cdot \nabla T-(\boldsymbol{\Pi}-p \mathbf{I}): \nabla \mathbf{v} \\
& -\mathbf{J} \cdot\left(\nabla \mu+\rho \alpha \frac{d^{*} \mathbf{J}}{d t}\right)-\mathbf{A}: \frac{d^{*} \mathbf{C}}{d t} \geq 0 .
\end{aligned}
$$

Note that in relation (21), the material time derivatives of $\mathbf{C}$ and $\mathbf{J}$ have been replaced by their corresponding Jaumann derivatives. This is justified because it is directly checked that $\mathbf{A}:(d \mathbf{C} / d t)=\mathbf{A}:\left(d^{*} \mathbf{C} / d t\right)$ and $\mathbf{J} \cdot(d \mathbf{J} / d t)=\mathbf{J} \cdot\left(d^{*} \mathbf{J} / d t\right)$ with $d^{*} \mathbf{J} / d t=d \mathbf{J} / d t+\mathbf{W} . \mathbf{J}$. To obtain a more pertinent expression for the entropy production rate, and to deduce the restrictions placed by the second law $\sigma^{s} \geq 0$, we need constitutive equations for the pressure tensor $\Pi$, the heat flux $\mathbf{q}$, or equivalently the entropy flux $\mathbf{J}^{s}$, and the evolution equation of $\mathbf{C}$. Taking into account their well-known expressions for molecular mixtures and suspensions $[5,11,16]$, together with simple symmetry and dimensional considerations, we are led to propose the following general expressions for $\boldsymbol{\Pi}$ and $\mathbf{J}^{s}$ :

$$
\begin{aligned}
\boldsymbol{\Pi}-p_{0} \mathbf{I} & =\boldsymbol{\tau}^{\mathrm{v}}+\boldsymbol{\tau}^{\mathrm{el}}+\gamma \mathbf{J} \mathbf{J}, \\
\mathbf{J}^{s} & =\frac{1}{T}\left[(\mathbf{q}-\mu \mathbf{J})-\delta \boldsymbol{\tau}^{\mathrm{v}} . \mathbf{J}\right],
\end{aligned}
$$

Expression (22) of the total pressure tensor is a generalization of the classical mechanical pressure tensor. $\boldsymbol{\tau}^{\mathrm{v}}$ and $\boldsymbol{\tau}^{\mathrm{el}}$ describe respectively the dissipative viscous and non-dissipative elastic mechanical forces, while the last term $\gamma \mathbf{J J}$ is associated with the mixing process with $\gamma$ being a phenomenological coefficient. Among these three contributions, only $\boldsymbol{\tau}^{\mathrm{v}}$ is classical; 
at equilibrium, $\boldsymbol{\Pi}$ reduces to $p_{0} \mathbf{I}$ as it should. Relation (23) of the entropy flux contains the classical contribution (the two terms between parentheses) and one extra term arising from the coupling of the fluxes and the pressure tensor; such a term is typical of the EIT formalism [10,12]. The evolution equation for the tensor $\mathbf{C}$ remains to be formulated. The dumbbell model [3] suggests to write it in the form

$$
\frac{d^{*} \mathbf{C}}{d t}=-\beta_{1} \mathbf{A}+\beta_{2} \mathbf{D}+\beta_{3}(\mathbf{D} . \mathbf{C}+\mathbf{C} . \mathbf{D}),
$$

with the quantity $\mathbf{R}$ introduced in Eq. (9) identified by the right-hand side of Eq. (24). A large indeterminacy is left in the above last three expressions, through the arbitrary scalars $\gamma, \delta$, and $\beta_{i}(i=1,2,3)$, which may depend on the density, the temperature, and the particle mass fraction (but not on $\mathbf{J}$ and $\mathbf{C}$ ).

With expressions (13), (16), and (22)-(24) taken for granted, the rate of dissipation $T \sigma^{s}$ finally appears in the standard form of a sum of products of fluxes and thermodynamic forces:

$$
\begin{aligned}
& T \sigma^{s}=-\boldsymbol{\tau}^{\mathrm{v}}: \nabla(\mathbf{v}+\delta \mathbf{J})+\beta_{1} \mathbf{A}: \mathbf{A} \\
&-\mathbf{D}: {\left[\boldsymbol{\tau}^{\mathrm{el}}+\beta_{2} \mathbf{A}_{0}+\beta_{3}\left(\mathbf{A}_{0} \cdot \mathbf{C}+\mathbf{C} \cdot \mathbf{A}_{0}\right)\right] } \\
&-\mathbf{J} \cdot\left\{\rho \alpha \frac{d^{*} \mathbf{J}}{d t}+\nabla \mu+\delta \nabla \cdot \boldsymbol{\tau}^{\mathrm{v}}+\gamma \mathbf{J} . \nabla \mathbf{v}\right. \\
&+\frac{1}{2} \alpha_{v}(\nabla \cdot \mathbf{v}) \mathbf{J}+\frac{1}{2} \rho \beta_{2}\left(\alpha_{\mathbf{C}}: \mathbf{D}\right) \mathbf{J} \\
&\left.+\frac{1}{2} \rho \beta_{3}\left[\left(\alpha_{\mathbf{C}} \cdot \mathbf{C}+\mathbf{C} \cdot \alpha_{\mathbf{C}}\right): \mathbf{D}\right] \mathbf{J}\right\} \\
&-\frac{1}{T}\left[(\mathbf{q}-\mu \mathbf{J})-\delta \boldsymbol{\tau}^{\mathrm{v}} \cdot \mathbf{J}\right] \cdot \nabla T \geq 0 .
\end{aligned}
$$

The viscous dissipation involves the gradients of the velocity $(\mathbf{v}+\delta \mathbf{J})$, suggesting that the following constitutive relation for $\tau^{\mathrm{v}}$ may be written:

$$
\boldsymbol{\tau}^{\mathrm{v}}=-\eta[\nabla(\mathbf{v}+\delta \mathbf{J})]^{\mathrm{sym}},
$$

with $\eta$ designating the viscous shear coefficient. The coupling between $\boldsymbol{\tau}^{\mathrm{v}}$ and $\mathbf{A}$ is prohibited; indeed, after substitution of the corresponding constitutive relations in Eq. (25), one should have obtained an expression of the entropy production that should be uneven with respect to time reversal, a result in contradiction with the fundamentals of thermodynamics [5,22]. 
Similarly, one observes in Eq. (25) the presence of a linear term in $\mathbf{D}$, by imposing that such a term will not contribute to the dissipation, it is inferred that the non-dissipative elastic tensor be given by

$$
\boldsymbol{\tau}^{\mathrm{el}}=-\beta_{2} \mathbf{A}_{0}-\beta_{3}\left(\mathbf{A}_{0} \cdot \mathbf{C}+\mathbf{C} \cdot \mathbf{A}_{0}\right) .
$$

From Eq. (22) and the results (26) and (27), we obtain the following expression for the tensor $\Pi$ :

$$
\boldsymbol{\Pi}-p_{0} \mathbf{I}=-\eta[\nabla(\mathbf{v}+\delta \mathbf{J})]^{\mathrm{sym}}-\beta_{2} \mathbf{A}_{0}-\beta_{3}\left(\mathbf{A}_{0} \cdot \mathbf{C}+\mathbf{C} \cdot \mathbf{A}_{0}\right)+\gamma \mathbf{J J} .
$$

Still, from expression (25) of the rate of dissipated energy, one is led to write for the heat flux and the evolution equation for the molecules diffusion flux the following expressions:

$$
\begin{gathered}
\mathbf{q}=-\lambda \nabla T+(\omega T+\mu) \mathbf{J}+\delta \boldsymbol{\tau}^{\mathrm{v}} \cdot \mathbf{J}, \\
\rho \alpha \frac{d^{*} \mathbf{J}}{d t}+\nabla \mu+\delta \nabla \cdot \boldsymbol{\tau}^{\mathrm{v}}+\gamma \mathbf{J} \cdot \nabla \mathbf{v}+\frac{1}{2} \alpha_{v}(\nabla \cdot \mathbf{v}) \mathbf{J}+\omega \nabla T+\xi \mathbf{J} \\
+\frac{1}{2} \rho \beta_{2}\left(\alpha_{\mathbf{C}}: \mathbf{D}\right) \mathbf{J}+\frac{1}{2} \rho \beta_{3}\left[\left(\alpha_{\mathbf{C}} \cdot \mathbf{C}+\mathbf{C} \cdot \alpha_{\mathbf{C}}\right): \mathbf{D}\right] \mathbf{J}=0 .
\end{gathered}
$$

In the case that configurational effects have negligible influence on diffusional transport (absence of added mass effect), the dependence of $\alpha$ with respect to $\mathbf{C}$ can be discarded and the two last terms in the left-hand side of Eq. (30) may be dropped. Concerning the coefficient $\alpha$, it is interesting to relate it to the relaxation time $\tau_{m}$ of the diffusion through

$$
\frac{\rho \alpha}{\xi}=\tau_{m}
$$

Just like $\xi$, the coefficients $\lambda$ and $\omega$ are phenomenological coefficients whose physical meaning will become clear later on. As a result of Eqs. (26)-(30), the dissipation inequality (25) becomes

$$
T \sigma^{s}=\eta \boldsymbol{\tau}^{v}: \boldsymbol{\tau}^{v}+\beta_{1} \mathbf{A}: \mathbf{A}+\xi J^{2}+\frac{\lambda}{T} \nabla T \cdot \nabla T \geq 0,
$$

from which it is inferred that the four dissipative coefficients $\beta_{1}, \xi$, $\eta$, and $\lambda$ are positive; no restriction is placed on the sign of $\omega$. Observe that, as expected, $\boldsymbol{\tau}^{\mathrm{el}}$ does not contribute to the dissipated energy $T \sigma^{s}$.

Besides $\xi$ and $\omega$, the evolution equation (30) of $\mathbf{J}$ depends on the following arbitrary parameters: 
- $\alpha$, a measure of the extra contribution in $J^{2}$ to the free energy,

- $\gamma$, introduced in expression (22) of the pressure tensor, and related to the $\mathbf{J J}$ contribution,

- $\delta$, associated with the mechanical contributions to the entropy flux (23),

- $\beta_{2}$ and $\beta_{3}$, reflecting the influence of the added mass effect.

A possibly interesting piece of information about some of these coefficients is obtained by comparing the above developments with internal variable theories $[14,15]$. One is then led to the following identifications:

$$
\rho \alpha=\gamma=\frac{1}{\rho c(1-c)}, \quad \delta=\frac{1}{\rho_{\mathrm{p}}}-\frac{1}{\rho_{\mathrm{c}}} .
$$

Introducing these values in Eq. (30), one recovers, at the exception of the terms involving $\beta_{2}$ and $\beta_{3}$, the same evolution equation for $\mathbf{J}$ as that obtained within the internal variable model $[14,15]$ by using the relative velocity $\left(\mathbf{v}_{\mathrm{p}}-\mathbf{v}_{\mathrm{c}}\right)$ as state variable.

In case all coefficients except $\xi$ and $\omega$ vanish, the evolution equation (30) boils down to

$$
\mathbf{J}=-\frac{1}{\xi} \nabla \mu-\frac{\omega}{\xi} \nabla T .
$$

Comparing the above relations with the classical Soret law [5], it is checked that $1 / \xi$ can be identified as the coefficient of diffusion and $\omega / \xi$ as the coefficient of thermal diffusion. For isothermal processes one recovers Fick's law $\mathbf{J}=-D_{\text {diff }} \nabla c$ with the diffusion coefficient defined by $D_{\text {diff }}=[(1 / \xi) \partial \mu / \partial c]_{T}$. In the absence of matter motion $\mathbf{J}=\mathbf{0}$, expression (29) of $\mathbf{q}$ leads to Fourier's law

$$
\mathbf{q}=-\lambda \nabla T,
$$

from which follows that $\lambda$ is the usual heat conductivity.

\section{More on the evolution equations of $\mathrm{J}$ and $\mathrm{C}$ and the constitutive relation of $\Pi$}

To clarify the meaning of the coefficients $\beta_{2}$ and $\beta_{3}$, let us consider a simplified expression of the general evolution equation (30) of $\mathbf{J}$. In the case that all the terms of Eq. (30) vanish with the exception of the second, third, and seventh, one is led to

$$
\mathbf{J}=-\frac{1}{\xi} \nabla \mu-\frac{\delta}{\xi} \nabla \cdot \boldsymbol{\tau}^{\mathrm{v}}
$$


which exhibits in a direct way the influence of the viscous dissipation stress tensor on the matter flow $\mathbf{J}$. A simple non-linear extension of Eq. (36) may be formulated by addition of the penultimate term of Eq. (30) involving the coefficient $\beta_{2}$ : within the linear approximation that $\alpha_{\mathbf{C}}$ is proportional to $\mathbf{C}$ and the assumption that $\mathbf{C}$ is proportional to $\mathbf{D}(\mathbf{C}=k \mathbf{D})$, this contribution is of the form $\rho k \beta_{2}(\mathbf{D}: \mathbf{D})$.J. Adding it to Eq. (36), one obtains

$$
\mathbf{J}=-\frac{1}{\xi^{*}} \nabla \mu-\frac{\delta}{\xi^{*}} \nabla \cdot \boldsymbol{\tau}^{\mathrm{v}},
$$

whose form is reminiscent of Eq. (36) but with a non-linear shear rate depending coefficient $\xi^{*}$ given by

$$
\xi^{*}=\xi+\rho k \beta_{2} \mathbf{D}: \mathbf{D} .
$$

According to wether $\beta_{2}$ is positive or negative will modify the influence of the mechanical rate of deformation. Keeping the last term in $\beta_{3}$ in the development (30) will be responsible of third order contributions in (D.D) : $\mathbf{D}$ to $\xi^{*}$. Interesting applications about the potential usefulness of the present work, and more specifically the results (36) and (37), are discussed in $[1,2,23]$.

Let us now comment upon the evolution equation of $\mathbf{C}$. Assuming as in Eq. (18) that $\mathbf{A}_{0}$ is proportional to $\left(\mathbf{C}-\mathbf{C}_{\mathrm{eq}}\right)$, the time evolution equation (24) of $\mathbf{C}$ can be cast in the form

$$
\frac{d^{*} \mathbf{C}}{d t}=-\frac{1}{\tau_{\mathrm{C}}}\left(\mathbf{C}-\mathbf{C}_{\mathrm{eq}}\right)+\beta_{2} \mathbf{D}+\beta_{3}(\mathbf{D} . \mathbf{C}+\mathbf{C} . \mathbf{D})-\frac{1}{2} \rho \beta_{1} \alpha_{\mathbf{C}} J^{2},
$$

which generalizes the results derived in Lhuillier and Ouibrahim [16] and Maugin [19] on different bases in the framework of the internal variables theory; $\tau_{\mathrm{C}}=r_{0}^{2} /\left(3 n k_{0} \beta_{1}\right)$ is the relaxation time of the conformation tensor and is checked to be a positive quantity as $\beta_{1}$ has been proven to be positive definite. In the particular case in which the identifications (33) hold, the evolution equation (39) assumes the simplified form

$$
\frac{d^{*} \mathbf{C}}{d t}=-\frac{1}{\tau_{\mathrm{C}}}\left(\mathbf{C}-\mathbf{C}_{\mathrm{eq}}\right)+\beta_{2} \mathbf{D}+\beta_{3}(\mathbf{D} . \mathbf{C}+\mathbf{C} . \mathbf{D}),
$$

and the pressure tensor will take the final form

$$
\Pi-p_{0} \mathbf{I}=-\eta(\nabla \mathbf{u})^{\mathrm{sym}}-\beta_{2} \mathbf{A}_{0}-\beta_{3}\left(\mathbf{A}_{0} \cdot \mathbf{C}+\mathbf{C} . \mathbf{A}_{0}\right)+\frac{1}{\rho c(1-c)} \mathbf{J J} .
$$


This result describes how to modify the constitutive equation for the stress tensor to include diffusion and particle deformation effects. It differs from the classical Newton's law of fluid mechanics by the presence of the three last terms in the right-hand side of Eq. (41). The second and third terms reflect the contribution of the deformed polymeric chains and the last term in $\mathbf{J J}$ arises as a consequence of the mixing process. Finally, observe that in contrast with Newton's law, the first term involves the gradient of the volume-weighted velocity $\mathbf{u}$ instead of the mass-weighted velocity $\mathbf{v}$. This result is well-known in the theoretical modeling of suspensions $[4,14,15]$. A last remark about Onsager's reciprocal relations [5] concerns form. Accordingly, the phenomenological coefficients of $\mathbf{D}$ in Eq. (40) and $\mathbf{A}_{0}$ in Eq. (41) must be the same but with a different sign, as one couples two "fluxes" $d^{*} \mathbf{C} / d t$ and $\boldsymbol{\Pi}-p_{0} \mathbf{I}$ of opposite parities with respect to time reversal; indeed, it is directly verified that the quantity $d^{*} \mathbf{C} / d t$ is uneven while $\Pi$ is even under time reversal: $t \rightarrow-t$. This indicates that the above description is in full agreement with Onsager's reciprocal relations and constitutes a supplementary argument in favor of the validity of the model.

\section{Final remarks}

The aim of this work was to propose a rather systematic analysis of deformable polymer transport in dispersed media based on non-equilibrium thermodynamics. The originality of the approach lies in enlarging the usual space of state variables, constituted by the global mass, mass fraction of polymers, temperature, and conformation tensor by the introduction of the matter diffusion flux $\mathbf{J}$. This attitude finds its roots in the developments of extended irreversible thermodynamics. Note, however, that in the present approach, only the flux of matter is elevated to the rank of state variable; other thermodynamic fluxes, like the pressure tensor $\Pi$ and the heat flux $\mathbf{q}$, are not considered as state variables but are instead given by constitutive relations. This was done for the sake of simplicity and because the relaxation times of these quantities are negligibly small in comparison with the relaxation time of $\mathbf{J}$.

Since the problem is not Fickean, the flux $\mathbf{J}$ will no longer satisfy the classical Fick's or Soret's laws but will obey a rather intricate time-evolution equation expressed by Eq. (30). Similarly, the heat flux $\mathbf{q}$ is not given by Fourier's law but obeys a more general expression (29). Finally, Newton's law of fluid mechanics is also generalized in the form (28) to 
comply with diffusion and rheological effects. Since it is required to make our formalism consistent with the principle of material frame indifference, the time evolution equation of the conformation tensor and the diffusion flux have been written in term of Jaumann's objective derivative.

The most important results of the present work are embodied in the evolution equations (30) and (39) of $\mathbf{J}$ and $\mathbf{C}$ and the constitutive equations (29) and (41) of the heat flow and total pressure tensor, respectively. According to Eq. (29), the heat flow is not only influenced by the temperature gradient but also by the mechanical and diffusive effects. Concerning the pressure tensor (41), it is shown to contain three contributions: the first is linked to the dissipative viscous effect, the second to the elastic non-dissipative forces, and the last to the mixing process.

It is true that the model implies knowledge of a great number of phenomenological coefficients. Some of them may be determined by comparison with other formalism like internal variable theories (see in that respect the identifications (33)); the remaining ones will be derived either from microscopic considerations and (or) experimental measurements. Observe that the number of the transport coefficients can be reduced by invoking Onsager's reciprocal relations.

As mentioned earlier, extended irreversible thermodynamics has been applied to several problems involving coupling of diffusion with internal structure (e.g., [9, 18]). More specifically, our evolution equation (30) of $\mathbf{J}$ can be considered as a refinement of the corresponding result obtained by Manero et al. [18]; indeed, their equation (22), which is the pendent of Eq. (30) above, is formulated under the assumption of uniform temperature and does not contain the terms involving the coefficients $\gamma, \alpha_{v}, \beta_{2}$, and $\beta_{3}$. Another difference lies in the choice of the internal variable describing the deformable particles, instead of the tensor $\mathbf{C}$, Manero et al. use a scalar variable $\zeta$. Constitutive equations for suspensions in polymer solutions have also been established though the GENERIC formalism (e.g., [7, 8, 20]). However, this theory which is based on a Hamiltonian formulation, departs rather radically from the above modeling and will therefore not be discussed further.

\section{Acknowledgments}

This work was partially supported by PRODEX (ESA) 2008-10 project and by University of Catania and University of Messina through P.R.A. The authors wish to thank the referees for their useful comments. 


\section{Bibliography}

[1] Agarwal, U. S., Dutta, A., Mashelkar, R. A., Migration of macromolecules under flow: The physical origin and engineering implications, Chem. Eng. Sci., 49 (1994), 1693-1717.

[2] Apostolakis, M. V., Mavrantzas, V. G., Beris, A. N., Stress gradient-induced migration effects in the Taylor-Couette flow of a dilute polymer solution, J. Non-Newtonian Fluid Mech., 102 (2002) 409-445.

[3] Bird, R. B., Curtiss, C. F., Armstong, R. C., Hassager, O., Dynamics of Polymeric Liquids, Kinetic Theory, Vol.2, Wiley, New York, 1987.

[4] Brenner, H., Rheology of two-phase systems, Ann. Rev. Fluid Mech., 2 (1970), 137-176.

[5] De Groot, S.R., Mazur, P., Non Equilibrium Thermodynamics, NorthHolland, Amsterdam, 1962.

[6] Francaviglia, M., Palumbo, A., Rogolino, P., Constitutive equations for internal variables thermodynamics of suspensions, J. Non-Equilib. Thermodyn., 34 (2009), 47-60.

[7] Grmela, M., Aitt-Kadi, A., Lafleur, J.P., Suspensions of fibers in viscoelastic fluids: Rheology, J. Chem. Phys., 109 (1998), 6973-6981.

[8] Gu, J.F., Grmela, M., GENERIC model of active advection, J. NonNewtonian Fluid Mech., doi: 10.1016/jnnfm 2007/ 05.001, (2007).

[9] Jou, D., Casas-Vázquez, J., Criado-Sancho, M., Thermodynamics of Fluids under Flow, Springer, Berlin, 2000.

[10] Jou, D., Casas-Vázquez, J., Lebon, G., Extended irreversible thermodynamics, 4th edn., Springer, Berlin, 2010.

[11] Landau, L., Lifshitz, E., Fluid Mechanics, Pergamon Press, New York, 1959.

[12] Lebon, G., Jou, D., Casas-Vázquez, J., Understanding Non-Equilibrium Thermodynamics, Springer, Berlin, 2008.

[13] Lebon, G., Lhuillier, D., Palumbo, A., A thermodynamic description of thermo-diffusion in suspensions of rigid particles, Eur. Phys. J. Special Topics, 146 (2007), 3-12.

[14] Lhuillier, D., From molecular mixtures to suspensions of particles, J. Phys. II France, 51 (1995), 19-36. 
[15] Lhuillier, D., Internal variables and the non-equilibrium thermodynamics of colloidal suspensions. J. Non-Newtonian Fluid Mech., 96 (2001), 19-30.

[16] Lhuillier, D., Ouibrahim, A., A thermodynamic model for solutions of deformable molecules, Journal de Mécanique, 19 (1980), 725-741.

[17] Lhuillier, D., Phenomenology of polymer migration, J. Phys. France, 44 (1983), 303-309.

[18] Manero, O., Perez-Lopez, J.H., Escalante, J.I., Puig, J.E., Bautisba, F., A thermodynamic approach to rheology of complex fluids: The generalized BMP model, J. Non-Newtonian Fluid Mech., 146 (2007), 22-29.

[19] Maugin, G., The Thermomechanics of Nonlinear Irreversible Behaviors, An Introduction, World Scientific, Singapore, 1999.

[20] Ottinger, H.C., Beyond Equilibrium Thermodynamics, Wiley, Hoboken, 2005.

[21] Truesdell, C., Rational Thermodynamics, 2nd edn., Springer, New York, 1984.

[22] Woods, L. C., Thermodynamics of Fluid Systems, Clarendon Press, Oxford, 1986.

[23] Zhen, J., Yeung, E.S., Mechanism for the separation of large molecules based on radial migration in capillary electrophoresis, Anal. Chem., 75 (2003), 3675-3680.

Received December 21, 2009; accepted January 29, 2010.

\section{Author information}

Annunziata Palumbo, Department of Mathematics, University of Messina, I 98166 Messina, Italy.

E-mail: apalumbo@unime.it (corresponding author)

Antonino Valenti, Department of Mathematics and Informatics, University of Catania, I 95125 Catania, Italy.

Georgy Lebon, Department of Astrophysics, Geophysics and Oceanography, University of Liège, B 4000 Liège, Belgium. 